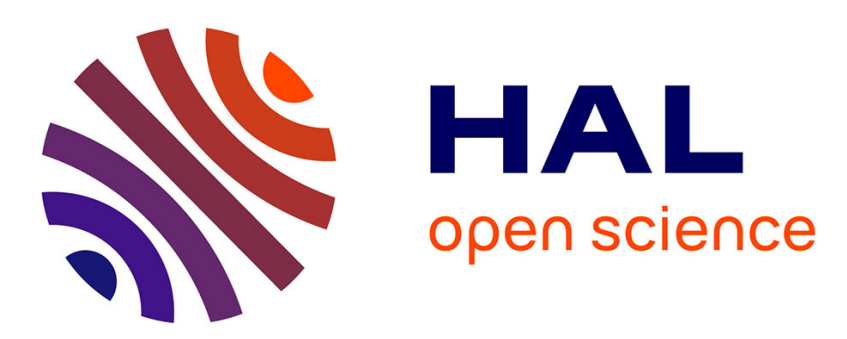

\title{
Mechanical Modelling of Capacitive RF MEMS Shunt Switches
}

\author{
Romolo Marcelli, A. Lucibello, G. de Angelis, E. Proietti, D. Comastri
}

\section{To cite this version:}

Romolo Marcelli, A. Lucibello, G. de Angelis, E. Proietti, D. Comastri. Mechanical Modelling of Capacitive RF MEMS Shunt Switches. EDA Publishing, pp.4, 2009. hal-00399326

\section{HAL Id: hal-00399326 \\ https://hal.science/hal-00399326}

Submitted on 26 Jun 2009

HAL is a multi-disciplinary open access archive for the deposit and dissemination of scientific research documents, whether they are published or not. The documents may come from teaching and research institutions in France or abroad, or from public or private research centers.
L'archive ouverte pluridisciplinaire HAL, est destinée au dépôt et à la diffusion de documents scientifiques de niveau recherche, publiés ou non, émanant des établissements d'enseignement et de recherche français ou étrangers, des laboratoires publics ou privés. 


\title{
DTIP \\ of MEMS \& MOEMS 1 1-3 April, Rome, Italy \\ Mechanical Modelling of Capacitive RF MEMS Shunt Switches
}

\author{
Romolo Marcelli, Andrea Lucibello, Giorgio De Angelis, Emanuela Proietti and Daniele Comastri \\ CNR-IMM Roma, Via del Fosso del Cavaliere 100, 00133 Roma, ITALY
}

\begin{abstract}
The mechanical response of a RF MEMS shunt connected switch in coplanar waveguide configuration has been studied by using both an analytical approach based on unidimensional equations and FEM simulations. The analytical model has been developed for predicting the dynamics of the device, especially concerning its re-configuration time and the energy balance involved in the actuation and in the de-actuation mechanisms. The analytical evaluation was based on a MATHCAD program. After that, $2 D$ and 3D simulations have been performed to get the deformed shape of the bridge, depending on the central and lateral actuation of the suspended beam. Theoretical and experimental results about the threshold voltage confirm the validity of the proposed modeling.
\end{abstract}

\section{ANALytical MODEL OF THE RF MEMS SWITCH}

RF MEMS switches are devices for signal processing at high frequencies, ideal candidates for substituting pin diodes in many applications, from redundancy switches, to phase shifters and matrices, even for space systems [1],[2],[3],[4]. They take advantage from being all passive devices, with no distortion and linear phase response. RF MEMS switches can be actuated in several ways but, generally, the electrostatic actuation is preferred because no current is flowing in the device and no power absorption is involved in the process.

The equation which accounts for the most part of the physical mechanisms governing the dynamics of a RF MEMS switch can be written as:

$m \ddot{z}=F_{e}+F_{p}+F_{d}+F_{c}$

where: $m=\rho A t$ is the bridge mass in the actuation region, computed by means of the density $\rho$, the area $A$ and the thickness $t ; F_{e}=\frac{1}{2} \frac{\partial C}{\partial z} V^{2}$ is the electric force due to the applied voltage $V$ and to the change of the capacitance along the direction of the motion $z ; F_{p}=-k[z-(d+g)]$ is the force due to the equivalent spring with its constant $k$, acting against the electrical force to carry back the bridge to the equilibrium position; $F_{d}=-\alpha \dot{z}$ is the damping of the fluid, dependent on the bridge velocity $\dot{z}$ and on the damping parameter $\alpha$, which, in turns, is related to the geometry of the bridge and to the viscosity of the medium, $d$ is the dielectric thickness and $g$ the gap between bridge and substrate. $F_{c}$ is the contact contribution, which can be divided in the Van Der Waals and surface forces, the first acting as attracting and the second one as repulsive, with a possible equilibrium position at a given distance from the bottom electrode of the switch [5]. Currently, many papers about the dynamics of the switch are available in literature (see, for instance, [10] and related references, or [3]), including also possible collateral effects due to the Casimir force [11],[12] or self-actuation mechanisms due to the RF power [13]. The capacitance of a shunt capacitive MEMS switch can be described in terms of two series capacitors, each of them having its own dielectric constant. From the above considerations, the total capacitance will be:

$C(z)=\frac{\varepsilon_{0} \varepsilon_{r} A}{d+\varepsilon_{r}(z-d)} ; \quad z \in[d, \quad d+g]$

where $\varepsilon_{0}=8.85 \times 10^{-12}$ is the vacuum dielectric constant in MKS units and $\varepsilon_{r}$ is the relative dielectric constant of the material covering the bottom electrode. The derivative of $C(z)$ is used in the definition of the electric force $F_{e}=\frac{1}{2} \frac{\partial C}{\partial z} V^{2}$ and Eq. (1) can be rewritten as:

$m \ddot{z}+k[z-(d+g)]+\alpha \dot{z}=-\frac{1}{2} \frac{\varepsilon_{0} \varepsilon_{r}^{2}}{\left[d+\varepsilon_{r}(z-d)\right]^{2}} V^{2}$

The voltage difference $V$ is imposed between the metal bridge, connected to the ground plane of a coplanar waveguide (CPW) structure, and the central conductor of the CPW, which also carries the high frequency signal. Under these circumstances, the switch will experience an electrostatic force for the actuation which is balanced by its mechanical stiffness, measured in terms of a spring constant $k$. The balance is theoretically obtained until the bridge is going down approximately $(1 / 3)$ of its initial height. After that, the bridge is fully actuated, and it needs a value of $V$ less than the initial one to remain in the OFF (actuated) position, because contact forces and dielectric charging effects help in maintaining it in the down position. The actuation as well as the de-actuation are affected also by the presence of a medium (typically air, or preferably nitrogen for eliminating humidity residual contributions in a packaged device) which contributes with its 
own friction, introducing a damping, and changing the speed of the switch [5],[6],[7]. Other contributions to the motion of the switch are given by the "contact force" of the bridge with respect to the plane, when it is actuated or very close to the plane of the CPW, i.e. close to the substrate. They are due to the interaction between the two surfaces. The Van Der Waals force, having an attractive effect, has to be also included [5],[7]. It is difficult to manage all of these contributions, and usually a phenomenological approach is followed, trying to individuate the most important parameters useful for the required response. For instance, higher is the ratio between bridge thickness and bridge length, higher will be the spring constant value and, consequently, the robustness of the switch. It is common practice that a good value for $k$ is in the order of tens of $\mathrm{N} / \mathrm{m}$. A recent theoretical evaluation is given in [14]

$k=\frac{2 E w t^{3}}{L_{d}^{3}}+\frac{E w \operatorname{tg}^{2}}{L_{d}^{3}}+\frac{2 \sigma(1-v) t w}{L_{d}}$

Where $L_{d}$ is half of the non-contact region of the bridge, defined as the full length of the bridge minus the actuation region. A MATHCAD program based on the above equations was written to predict the actuation and de-actuation of the switch. Typical responses for the de-actuation envelope curve of a RF MEMS switch are given in the following Fig. 1, where the contributions of the restoring mechanical force and of the damping have been clearly evidenced. For the results of Fig. 1 as well as for the next ones, we assume the following parameters for the simulated bridge: $L=600 \mu \mathrm{m}$ as the bridge total length, $L S=300 \mu \mathrm{m}$ as the switch length in the RF contact region (width of the central conductor of the CPW), $w=100 \mu \mathrm{m}$ as the bridge width, before the contact region, to be averaged as an approximation of the real cases if a tapering is present, $w S=100 \mu \mathrm{m}$ for the switch width (transversal dimension of the switch, parallel with respect to the CPW direction), $d=$ thickness of the dielectric material $=0.1 \mu \mathrm{m}$, with dielectric constant $\mathcal{E}=3.94\left(\mathrm{SiO}_{2}\right), t=1.5 \mu \mathrm{m}$ for the gold bridge, $\rho=19320$ $\mathrm{kg} / \mathrm{m}^{3}$ for the gold density, $E=$ Young modulus $=80 \times 10^{9} \mathrm{~Pa}$, $v=0.42$ for the metal Poisson coefficient and $\sigma=18 \mathrm{MPa}$ as the residual stress of the metal.

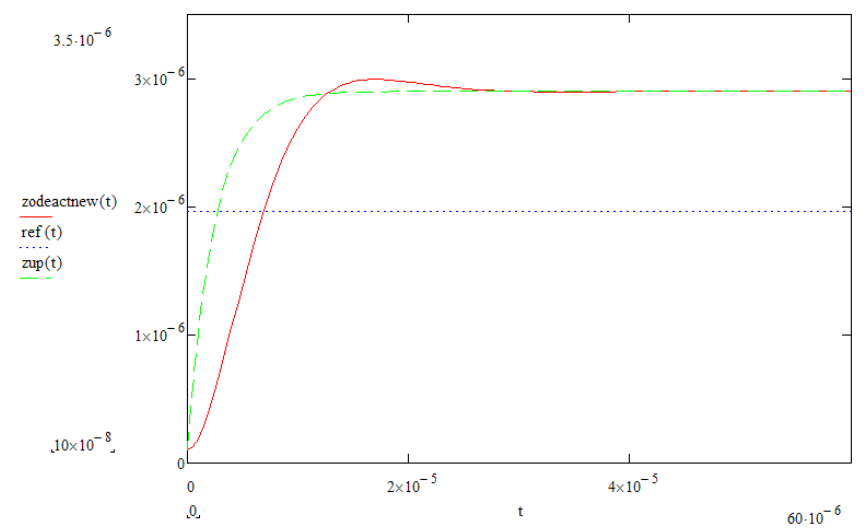

Fig. 1. Envelope of the de-actuation Vs time for the shunt switch used as an example (MATHCAD computation). The green curve is the exponential

envelope of the restoring mechanism, while the red one accounts also for the air damping. The reference line is drawn at $(2 / 3)$ of the full gap.
1-3 April, Rome, Italy

From capacitance dynamics considerations at the threshold voltage, it can be assumed that the de-actuation time is defined as the time at which the capacitance reaches the $90 \%$ of the final one. By using a purposely developed analytical treatment based on energy considerations, the actuation time has be obtained from the following equation:

$\tau_{\text {deact }}=-\frac{2}{\beta} \ln \left[\frac{1}{9}\left(1-\frac{d}{\varepsilon g}\right)\right] \quad \tau_{\text {act }}=-\frac{2}{\beta} \ln \left(\frac{1}{9} \frac{d}{\varepsilon g}\right)$

Then, we included the dependence on the applied voltage, obtained by means of the energy spent for the actuation, and the second of Eq. (4) was transformed in:

$\tau_{a c t}=-\frac{1}{\beta} \ln \left(\frac{4}{\beta^{2} g^{2}} \frac{C_{O F F} V_{a}^{2}-k g^{2}}{m+2 \frac{\beta}{\omega}}\right)$

Where $C_{O F F}$ is the capacitance in the down state, and it has been also given the explicit dependence on the applied voltage $V_{a}$. In Fig. 2 the actuation time Vs the applied voltage is shown.

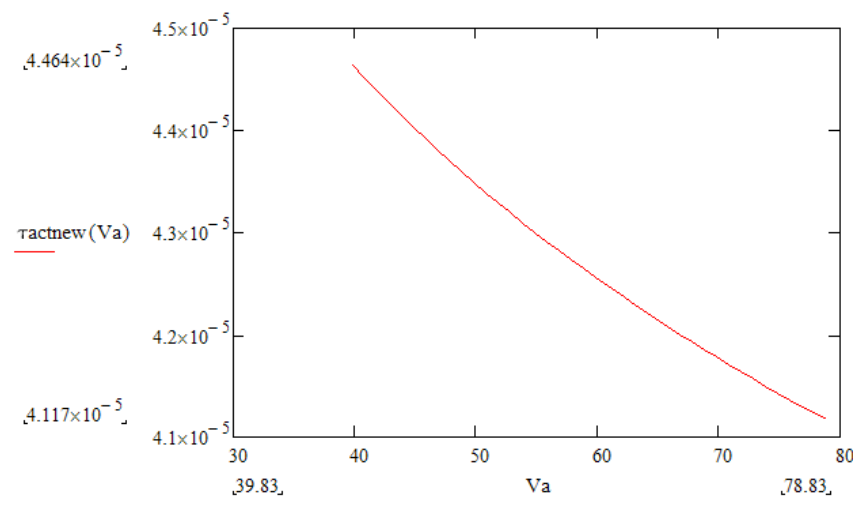

Fig. 2. Actuation time (in sec) of an RF MEMS capacitive shunt switch Vs the applied voltage $V_{a}$ (in volt).

The results in Fig. 2 are confirmed by those in Fig. 3, where the actuation dynamics calculated by the analytical approach is shown, by imposing the voltage threshold for this configuration, i.e. $V_{\text {threshold }} \approx 40$ volt, calculated by means of the definition in [3].

\section{BI- AND ThreE-DimenSIONAL MeChaniCAL SIMULATIONS}

$2 \mathrm{D}$ and $3 \mathrm{D}$ approaches have been used to simulate the same structure, based on the software package COMSOL Multiphysics. Simple geometries can be efficiently simulated by using an uni-dimensional approach, as also stated in [3], and actuation times can be estimated without using long and complicated simulations with finite element methods (FEM). On the other hand, a full simulation is very important when the shape of the bridge is tailored in a not simple way. This happens when the cross section has not a constant width, or specific technological solutions, like multi-layers for the bridge and dimples are realized in the actuation area, to improve the electrical contact; or holes are present on the beam to help the sacrificial layer removal and to lower the spring constant. 


\section{DTIP}

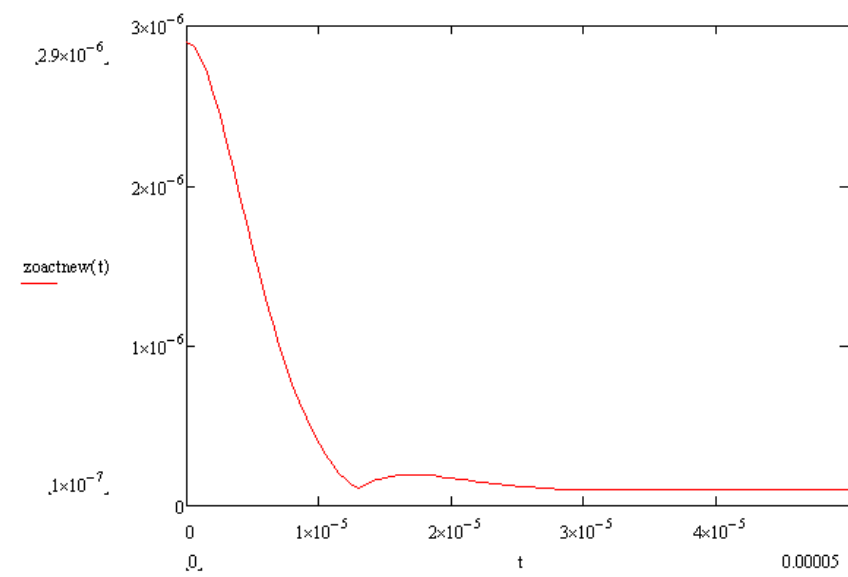

Fig. 3. Actuation of the exploited bridge. The complete OFF state is obtained starting from $40 \mu \mathrm{s} \mathrm{ca}$. from the application of the force. The damped oscillation is due to the analytical solution of the motion equation, as also evidenced in Fig. 1, corresponding to a possible bounce before the full actuation of the bridge.

For the above situations, only effective quantities can be defined in an analytical approach. Moreover, a software to treat combined solicitations involving the mechanical, thermal and electrical response of the MEMS device could be useful in the definition of a figure of merit for such a technology. For this purpose, 2D and 3D mechanical simulations have been performed to clearly state the advantages of such an approach with respect to the uni-dimensional one. Some 2D results are presented in Fig. 4 and in Fig. 5, where the OFF state of the switch is shown by using a central actuation (DC signal along the central conductor of the $\mathrm{CPW}$ ) or a lateral one by means of symmetrical pads (RF and DC paths separation).

The proper shape of the actuated bridge, also in the case of simple geometries, is particularly useful for the prediction of the electrical properties of the device. In fact, the real deformed shape of the bridge can be used as the input for high frequency simulations, and parasitic contributions could be predicted in an easier way, especially when the millimetre wave range is considered.

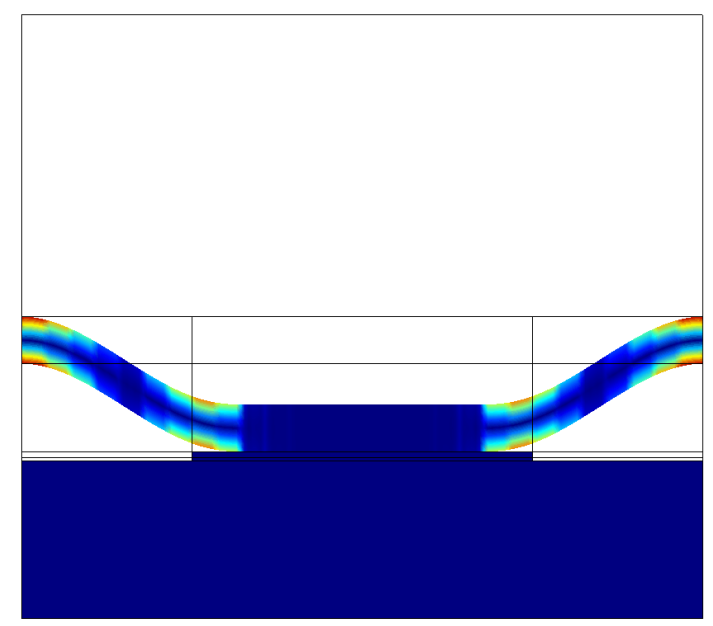

Fig. 4. 2D simulation for the central actuation of the MEMS switch (COMSOL simulation). A mechanical force on the central conductor of the CPW has been applied as high as $9000 \mathrm{~N} / \mathrm{m}^{2}$ to obtain an almost full actuation. In this case, the computation is performed including the width of the bridge just as a parameter.
1-3 April, Rome, Italy

From the result in Fig. 5, it turns out that by properly choosing the shape and the dimensions of the structure, the actuation occurs without having the bridge touching the lateral pads. This could help in decreasing the charging effects for these devices, mainly due to the dielectric used onto the actuation pads. The necessity to separate the RF and DC paths is the reason for preferring a lateral actuation in actual devices.

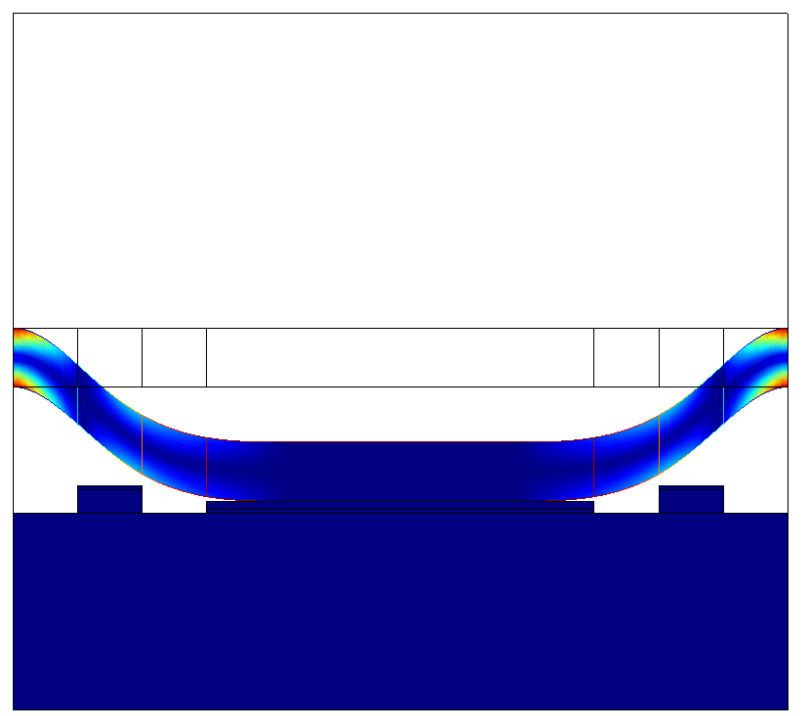

Fig. 5. Lateral actuation of the $2 \mathrm{D}$ structure (COMSOL simulation). The same force was necessary for having a full collapse of the bridge, but applied on smaller lateral pads (50 $\mu \mathrm{m}$ width). A more uniform actuation is obtained, as evidenced by the colour in the central part. Moreover, it is contact-less on the pads, which helps in minimizing the charging effects.

An example of the 3D response is given in the following Fig. 6 and Fig. 7, where the actuation has been performed by means of the same force used for the $2 \mathrm{D}$ case, with details about the full device.

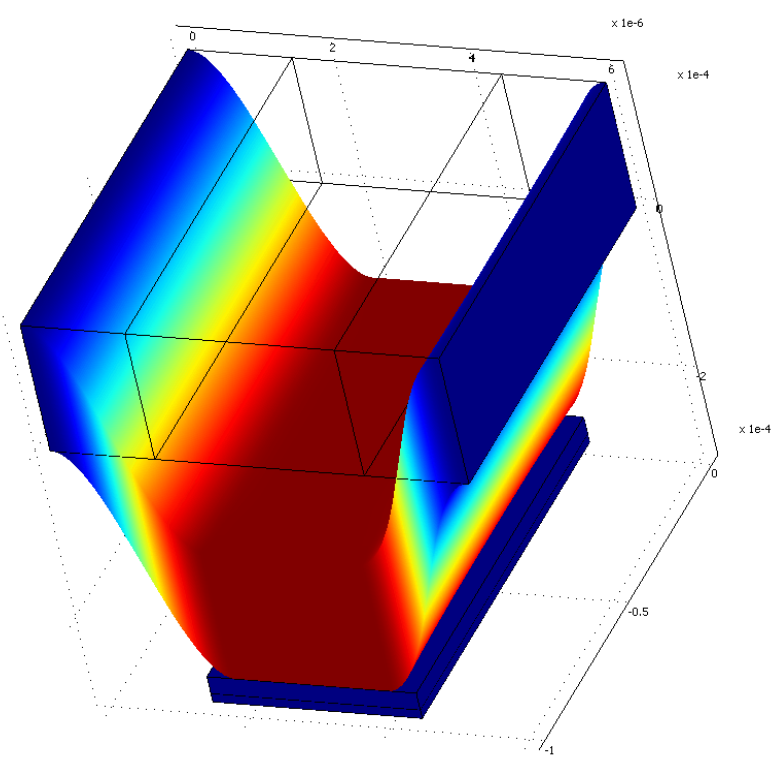

Fig. 6. 3D COMSOL simulation of the RF MEMS shunt capacitive switch in the OFF state (bridge in the down position), centrally actuated.

The result in Fig. 6 is coherent with the prediction performed by using the analytical approach, as the imposed mechanical 
force corresponds to an applied voltage $V_{a}=40$ volt, thus demonstrating that the actuation of the bridge can be easily predicted by using the uni-dimensional approach, exception done for those structures where holes or shape tailoring are present.

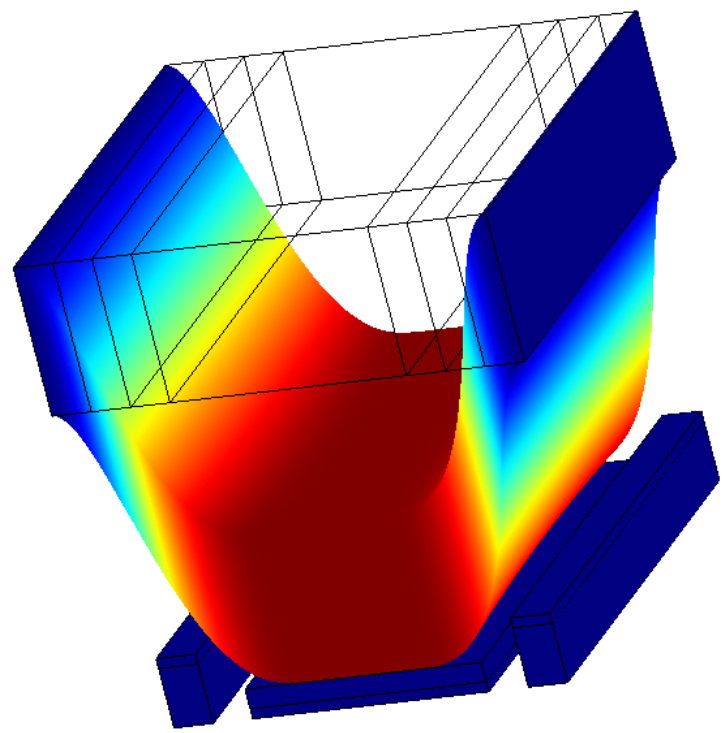

Fig. 7. 3D simulation of the RF MEMS switch in the OFF state (bridge in the down position), laterally actuated when the applied force is $\mathrm{F}=9000 \mathrm{~N} / \mathrm{m}^{2}$. The deformation of the bridge is represented by the change in the colours, from the

blue (at rest) to the red (fully actuated). The distribution of the force in the actuation area is more uniform with respect to the case of Fig. 5.

Only in simple cases the presence of holes can be approximated by defining an effective stiffness for the metal beam. For the above reason, the 3D simulation is really useful, as already stressed, only in the case of configurations which have a very peculiar shape. Also in the case of a moving mesh, to be used for the dynamical response of the device, many information can be already obtained from evaluations based on the previous equations, by using a fully analytical model, without involving cumbersome simulations with a computer. The real advantage in having a full modelling of the device is in the combination of mechanical and RF predictions, being based on the same geometry construction. Specifically, as it is the case of the COMSOL software package, thermal, power and charging effects can be considered in the same simulation environment. For the above reasons, this will be very useful to get a figure of merit for the RF MEMS technology based on different input conditions.

\section{EXPERIMENTAL RESULTS}

An actual configuration having the same dimensions described in the previous sections has been realized and preliminary tested. A photo of the structure, using a central actuation, is given in Fig. 8. SU-8 polymeric sides have been realized by photo-lithography to obtain the ground planes of the CPW, where the metal bridge is suspended. Silicon oxide has been deposited as a dielectric, and the actuation has been performed by means of the central conductor of the CPW. Actuations in the order of 30-35 volt have been obtained in different samples, as expected accounting also for the presence
1-3 April, Rome, Italy

of the holes, which lower the stiffness of the beam with respect to the ideal case ( $V_{\text {threshold }}=40$ volt for a uniform beam).

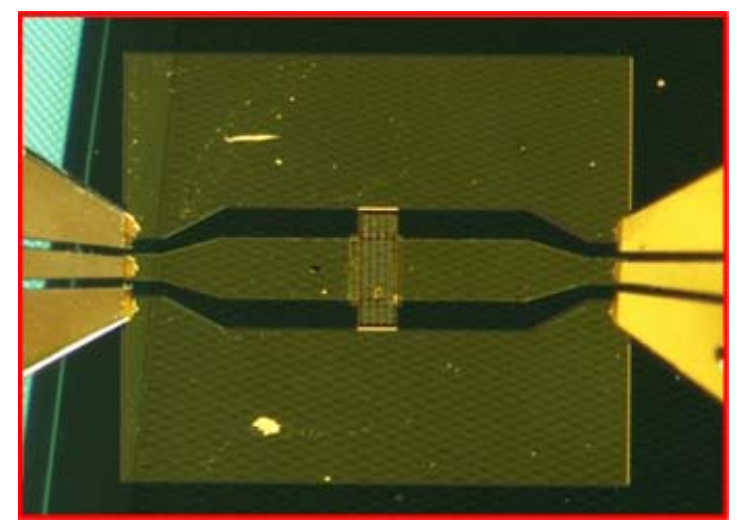

Fig. 8. RF MEMS switch realized by means of SU-8 photo-lithography.

ACKNOWLEDGEMENT

The authors wish to acknowledge the funding from the Contract ESA/ESTEC AO 1-5288/06/NL/GLC: High Reliability MEMS Redundancy Switch.

\section{REFERENCES}

[1] G. M. Rebeiz and J. P. Muldavin: "RF MEMS Switches and Switch Circuits", IEEE Microwave Magazine, Vol.2, No.4, pp.59-71 (2001).

[2] G. M. Rebeiz, Guan-Leng Tan and J. S. Hayden: "RF MEMS Phase Shifters, Design and Applications", IEEE Microwave Magazine, Vol.3, No.2, pp.72-81 (2002)

[3] G. M. Rebeiz, "RF MEMS, Theory, Design and Technology", John Wiley and Sons, Hoboken, 2003.

[4] Harrie A.C. Tilmans: "MEMS components for wireless communication", invited paper at XVI Conference on Solid State Transducers, Prague, Czech Republic, Sept. 15-18, 2002

[5] E.K. Chan, E.C. Kan and R.W. Dutton: "Nonlinear Dynamic Modeling of Micromachined Switches", Proceed. Of IEEE MTT-Symposium, pp.15111514 (1997).

[6] E.K. Chan and R.W. Dutton: "Effect of Capacitors, Resistors and Residual Charge on the static and dynamic Performance of Electrostatically Actuated Devices", SPIE Symposium on Design and Test of MEMS/MOEMS - March 1999.

[7] D. Mercier, P. Blondy, D. Cros and P. Guillon: "An Electromechanical Model for MEMS Switches", Proceed. Of IEEE MTT-Symposium, pp.2123-2126 (2001).

[8] J. B. Muldavin and G. M. Rebeiz, "Nonlinear Electro-Mechanical Modeling of MEMS Switches", Proceed. of IEEE MTT Symposium, pp.21119-2122 (2001).

[9] Fuqian Yang, "Electromechanical Instability of Microscale Structures", $J$. Appl. Phys, Vol. 92, No. 2, pp.2789-2794 (2002)

[10] Z. J. Guo, N. E. Mc Gruer and G. G: Adams, "Modeling, simulation and measurement of the dynamic performance of an ohmic contact, electrostatically actuated RF MEMS switch", J. Micromech Microeng., Vol. 17, pp.1899-1909 (2007)

[11] F. Michael Serry, Dirk Walliser and G. Jordan Maclay, "The role of the Casimir effect in the static deflection and stiction of membrane strips in microelectromechanical systems (MEMS)", J. Appl. Phys., Vol. 84, No. 5 pp. 2501-2506 (1998)

[12] Steve K. Lamoreaux, "Casimir Forces: Still surprising after 60 years", Physics Today, February 2007, pp.40-45 (2007)

[13] Karl M. Strohm et al., "RF-MEMS Switching Concepts for High Power Applications", Proceed. of 2001 IMS, pp.42-46 (2001).

[14] Balaji Lakshminarayanan, Denis Mercier, and Gabriel M. Rebeiz "HighReliability Miniature RF-MEMS Switched Capacitors", IEEE Trans. on Microwave Theory and Tech., Vol.56, No. 4, pp.971-981 (2008). 